Annuaire suisse de politique de développement

$21 \mid 2002$

Agriculture suisse et mondialisation

\title{
Un autre modèle de développement agricole dans le Sud est-il possible?
}

\section{Claude Auroi}

\section{(2) OpenEdition}

1 Journals

Édition électronique

URL : http://journals.openedition.org/aspd/932

DOI : 10.4000/aspd.932

ISSN : 1663-9669

Éditeur

Institut de hautes études internationales et du développement

\section{Édition imprimée}

Date de publication : 1 avril 2002

Pagination : 91-105

ISSN : 1660-5934

\section{Référence électronique}

Claude Auroi, « Un autre modèle de développement agricole dans le Sud est-il possible ? », Annuaire suisse de politique de développement [En ligne], 21 | 2002, mis en ligne le 06 septembre 2012, consulté le 08 septembre 2020. URL : http://journals.openedition.org/aspd/932 ; DOI : https://doi.org/10.4000/ aspd.932 


\title{
UN AUTRE MODÈLE DE DÉVELOPPEMENT AGRICOLE DANS LE SUD EST-IL POSSIBLE?
}

\author{
Claude Aurol*
}

$L$

ES TRANSFORMATIONS MONDIALES ont profondément affecté les économies et sociétés rurales depuis une vingtaine d'années. Les nouvelles politiques de développement agricole mises en place dans le cadre des programmes d'ajustement structurel et d'ouverture du commerce mondial n'ont pas apporté de solutions satisfaisantes à la situation des agricultures du Sud. De nouveaux principes de développement devraient être formulés qui permettent d'articuler organiquement le secteur agraire avec les économies nationales et de concevoir l'exportation et les aides extérieures comme des compléments, non comme des fondements. Pour cela, le concept «utiliser nos propres ressources locales » peut contribuer à faire avancer la réflexion des décideurs.

Nous décrirons tout d'abord les changements qui sont apparus au passage d'un système à l'autre.

\section{L'ANCIEN SYSTÈME}

Les politiques agricoles internationales et nationales se sont beaucoup modifiées depuis les années 1980, dans le cadre d'un changement plus global de paradigme: la concurrence au lieu de la protection.

Depuis la fin de la Seconde Guerre mondiale et la mise en place des institutions internationales de développement, l'agriculture a été considérée comme un secteur à développer dans le cadre de stratégies nationales des pays en développement. Ce développement était une affaire de l'Etat, qui devait gérer des instruments d'organisation de la paysannerie (coopératives), d'encadrement des producteurs (services techniques), de fourniture des intrants (sociétés d'Etat), et des services de recherche et de vulgarisation (instituts de recherche agronomique et services de la vulgarisation).

La commercialisation des produits devait aussi être prise en charge par des services d'Etat ou para-étatiques, surtout lorsqu'il s'agissait de cultures de rente exportables. Le financement de la production et la régulation des prix étaient gérés en Afrique par des systèmes de marketing boards ou «offices de commercialisation et caisses de stabilisation», qui permettaient théoriquement de se garantir contre les fluctuations des prix internationaux et des taux de change.

Au port d'embarquement du cacao, du sucre ou du café, c'étaient alors des sociétés internationales spécialisées qui prenaient en charge la commercialisation des produits sur le plan international. Les Etats du Sud n'avaient pas ou peu de participation dans ces sociétés. En fait, tout le système constituait en une 
sorte de poursuite des agricultures coloniales de plantation, permettant d'une part de maintenir le pouvoir des grandes sociétés céréalières, de produits tropicaux et de matières premières (coton), et de l'autre de contenter en partie des bourgeoisies locales en formation cherchant à s'approprier une portion des hypothétiques plus-values de l'exportation. Dans ce panorama, les petits producteurs n'étaient considérés comme partenaires que s'ils pouvaient produire pour le marché international, ou étaient à la base de quelques grandes cultures de subsistance - riz en Asie, maïs en Amérique centrale et du Sud, maïs et manioc en Afrique, pommes de terre dans les Andes -, ou, dans certaines régions appropriées, pratiquaient l'élevage.

Mais les soutiens allaient en priorité aux grands et moyens producteurs, sous forme de prêts à des taux d'intérêt préférentiels, de facilités douanières d'importation des intrants et machines, et de subventions à la production d'intrants locaux (engrais azotés et phosphatés). Dans ce processus, le petit paysan n'a que rarement été pris en compte, et cela malgré de multiples programmes d'aide internationaux et un grand travail des ONG. Dès lors, si le petit paysan a progressé, ce qui est difficile à évaluer, c'est par ses propres efforts, ses stratégies de survie et sa débrouillardise (Haubert 1997). Pendant longtemps les petits paysans d'une communauté rurale n'avaient pas les capacités intellectuelles (analphabétisme), techniques, organisationnelles et financières, ni même souvent l'envie de se lancer dans la production agricole moderne et marchande.

Dans les années 1960-1970, leur intégration aux marchés était soit inexistante, soit restait en dessous de $25 \%$ de leur produit. Mais elle s'est progressivement intensifiée et vingt ans plus tard, la production pour le marché atteint presque partout entre $30 \%$ et $80 \%$ (Auroi et Maurer 1998). Les facteurs de ce changement tiennent plus à la capacité de mobilisation du paysannat qu'aux aides qui lui furent octroyées. La croissance des villes, la motorisation, l'extension et l'amélioration des routes ainsi que la progression de la scolarisation ont créé un autre type de paysan, qui effectivement a peu à peu cherché à s'intégrer au marché - pour se heurter à des obstacles qu'il n'avait pas prévus.

\section{LA CRISE DES ANNÉES 1980}

Lorsque, vers le milieu des années 1980, la paysannerie de bon nombre de pays latino-américains et de certains pays africains aurait pu devenir un fournisseur privilégié de denrées alimentaires pour le marché interne, déplaçant les importations, elle s'est trouvée confrontée à l'amorce du changement du modèle traditionnel, changement dû à la répercussion de la crise internationale. Le moratoire mexicain sur la dette de 1982, le moratoire péruvien de 1985, les difficultés de remboursement des pays africains ont entraîné le changement d'orientation des politiques agricoles officielles. Les programmes d'ajustement structurel, ou PAS, furent alors introduits.

De fait, les PAS signifiaient «moins d'aide de l'Etat à l'agriculture », dans une perspective néolibérale de «laisser-faire» pour retrouver les avantages compétitifs. Les produits compétitifs sur les marchés internationaux seront privilégiés, les autres abandonnés, et la paysannerie devra se reconvertir. Le sous-secrétaire mexicain à la planification agricole disait en 1991 que «la politique déclarée de 
mon gouvernement est de diminuer de moitié la population du Mexique rural dans les cinq prochaines années » (cité par Barkin 2000).

Le désengagement de l'Etat a dès lors touché fortement une paysannerie montante, qui s'est trouvée démoralisée. Les coopératives et groupes villageois en Afrique ont été abandonnés ou dissous au profit soit de groupes d'intérêts économiques (GIE), rassemblant essentiellement des producteurs « rentables», soit de nouveaux ruraux, formés de fonctionnaires «déflatés » ${ }^{1}$ et de commerçants ${ }^{2}$.

Le soutien par subventions, aux intrants et aux machines, ainsi que les tarifs douaniers préférentiels ont été supprimés, les fabriques d'engrais et de matériel agricole fermées. Dans beaucoup de cas, cela signifia soit que seuls les paysans riches et les fonctionnaires purent encore avoir accès à ces services, soit que les technologies agraires rétrocédèrent. Le cas le plus typique fut l'abandon de Cuba par la Russie, ce qui entraîna une pénurie de pétrole, la paralysie des tracteurs et charrues, et le retour au labour par l'araire, la coupe de la canne à la machette.

Les services techniques de 1'Etat et les services de recherche et vulgarisation ont été soit réduits à la portion congrue, soit supprimés. Dans beaucoup de pays, le crédit agricole, déjà réservé aux paysans moyens et riches, a été supprimé et transféré au secteur privé.

L'idée de base était en effet de privatiser tous les services qui pouvaient l'être, en estimant que le secteur privé est de toute manière plus efficace que l'Etat. De fait, derrière cette appréciation officielle il faut voir deux phénomènes : l'un tient à l'endettement des Etats et aux pressions qui furent exercées sur eux par les institutions financières internationales qui les secouraient, et l'autre à la pression des grands groupes opérant dans le secteur agraire (Massey-Fergusson, Fiat, Bayer, Ciba-Geigy) qui cherchèrent à s'approprier tout le secteur des intrants.

On nota le même phénomène de démantèlement de l'influence de l'Etat dans la commercialisation des produits. Du côté des exportations de produits tropicaux, les systèmes de caisses de stabilisation des prix (marketing boards) furent dissous en Afrique, les sociétés de commercialisation abandonnées en Amérique latine, et si l'Asie résista, ce fut parce que l'Etat s'opposa à leur disparition, et surtout qu'il avait encore des moyens financiers, du moins jusqu'à la crise financière thaï (1997), puis indonésienne, malaise et coréenne. En réalité, les grands groupes exportateurs dans ce secteur jouaient sur du velours, du fait d'une certaine surproduction de café, de cacao et d'autres produits, surproduction que les groupes avaient eux-mêmes encouragée pendant au moins deux décennies. En raison de la stagnation des marchés extérieurs, les recettes des Etats diminuèrent encore, alors qu'au cours des discussions de l'Uruguay Round (1994) on fit miroiter aux pays exportateurs un meilleur accès à ces marchés. Cela ne se produisit pas car les pays du Nord n'ouvrirent que timidement leurs marchés, généralement aux pays les moins avancés, qui de toute façon avaient peu à offrir qu'ils n'exportaient déjà. Un exemple parfait est donné par le Burkina, qui, à part le coton, et le bétail (pour l'Afrique côtière), ne peut pas vraiment espérer exporter de grandes quantités de haricot vert ou de mangues (Aly Bâ 2001).

1. «Déflaté » : terme utilisé en Afrique francophone pour désigner les fonctionnaires licenciés.

2. En Afrique, la diminution des emplois publics n'a pas été entièrement comblée par une augmentation de l'emploi informel et rural. Cf. BIT (1999). 
Il y eut stagnation des recettes d'exportation, mais par contre importations accrues de produits du Nord dans les marchés du Sud. Cette tendance n'est pas vraiment nouvelle, car les exportations de céréales ont été subventionnées aux Etats-Unis dès les années 1950, dans le cadre du soutien des producteurs du Middle West et de la lutte contre le communisme international (Public Law $\left.480^{3}\right)$. L'Europe, surtout depuis qu'elle est devenue Marché commun, puis Union, a emboîté le pas des subventions des exportations, au vu de surplus de production toujours croissants. Or cette surproduction est le résultat direct d'encouragements importants de la politique agricole commune, qui a cherché à freiner le mécontentement paysan, ainsi qu'à concurrencer les Etats-Unis par «l'arme alimentaire» (Bessis 1985) sur les marchés extérieurs stratégiques. Mais les exportations de céréales vers le Sud ainsi que celles de viande dépriment souvent la production locale, changent les modèles alimentaires locaux, accroissent la dépendance alimentaire de l'extérieur, augmentent la charge de la balance commerciale et contribuent à faire régner l'insécurité de l'approvisionnement. L'exemple mexicain est clair sur tous ces points. La suppression des aides aux granos basicos (haricots et maïs) dans les années 1970 a entraîné l'importation de maïs des Etats-Unis, mais aussi de plus de blé, le recul du frejol (haricot) dans la cuisine nationale, et l'appauvrissement des paysans producteurs (Feder 1978; Barkin et Suárez 1982). Dans le cas du Sénégal, la substitution du mil par le riz a déjà commencé sous la colonie, mais par la suite même le riz local de Casamance a été concurrencé par des riz asiatiques, et les grands investissements hydrauliques dans la riziculture de la vallée du Sénégal ne sont guère rentables du fait du prix très bas des importations.

\section{LE PROBLÈME DE LA TERRE}

Dans la doctrine de la libéralisation, la terre est considérée comme une marchandise, bien que d'un type spécial. C'est également un actif (asset), et c'est justement pour cela qu'elle devrait pouvoir être négociée sans entraves, vendue, achetée, louée, mise en gage. Dès lors la stratégie néolibérale a consisté à privatiser le plus possible la terre, en supprimant les formes de propriété collective, surtout lorsqu'elles étaient inaliénables par le droit coutumier. On a ainsi au Mexique la loi de dissolution des ejidos de 1991, au Pérou la levée de l'interdiction de vente de terre des communautés indiennes (1993). En Afrique, on assiste aussi à une tendance à la privatisation, à la suppression de la fonction de «maître des terres» dans les villages sahéliens, à la concession de terres aux néoruraux.

Si cette tendance à la privatisation est bien réelle et qu'elle s'est inscrite dans des lois, elle n'a heureusement pas déployé tous les effets néfastes qu'on aurait pu craindre.

3. La PL 480 jouit de la définition officielle suivante (site Internet de l'USAID <www.usaid.gov/pubs/ cp2000/pl480ffp.html>): «U.S. support for overseas food aid was formalized in the Agricultural Trade Development and Assistance Act of 1954, also known as P.L. 480 Food for Peace. The basic legislation, which has been modified many times, establishes the U.S. policy of using the country's abundant agricultural resources and food processing capabilities to enhance food security in the developing world through the provision of culturally acceptable nutritious food commodities. 》 
Les quelques études dont nous disposons dans les cas mexicain et péruvien, mais aussi dans les cas de l'Equateur et de la Bolivie, montrent que les lois de privatisation n'ont pas entraîné de ventes massives, ni même souvent de ventes tout court (Mayer 2002 ${ }^{4}$ ). Les communautés n'ont pas été non plus dissoutes, et les habitants n'ont pas quitté la terre d'une manière accélérée. Sous l'influence d'un indigénisme grandissant en Amérique latine, de l'organisation toujours plus poussée grâce aux moyens modernes de communication, de l'apparition de leaders paysans et indigènes toujours mieux formés, la valeur du «collectif» a au contraire augmenté, spécialement dans les zones à forte proportion de population indienne.

Ce mouvement est relayé par les pressions internationales concernant la protection de la biodiversité et des ressources naturelles en général, par la création de parcs nationaux et régionaux, et par l'écotourisme. Il apparaît alors souvent que la meilleure manière de protéger la terre et les ressources est de le faire à travers la propriété communale, l'indivis et la restriction à l'appropriation privée des ressources.

Enfin, si l'on avait pu craindre une ruée sur les nouvelles terres négociables, les grandes compagnies n'ont pas manifesté ce mouvement, ni les grands propriétaires. Cela est probablement dû au fait que les terres communautaires sont parmi les plus mauvaises, pentues et en altitude, et donc peu attractives. Il est aussi d'autres moyens pour faire travailler la terre que la propriété: les contrats de livraison, la sous-traitance, etc. Il y a plusieurs décennies que les grandes compagnies bananières opérant en Amérique centrale ne possèdent plus directement les plantations. Le contrôle du commerce de la banane est beaucoup plus intéressant au niveau des profits que la production directe, elle-même source de conflits du travail incessants. Les multinationales laissent donc les propriétaires locaux et l'Etat s'occuper de l'organisation de la production, n'intervenant qu'au niveau des normes techniques et de qualité, dans certains cas de la fourniture de semences ou boutures sélectionnées.

\section{DÉRÉGULATION PROVENANT DES ACCORDS DE MARRAKECH}

On aurait pu penser que les accords créant l'OMC en 1994 allaient aggraver la situation des paysanneries du Sud, car les protections devaient tomber aussi au Sud, bien que plus modérément et dans un délai plus long. Les pays les moins avancés étaient même exemptés de prendre des mesures de détarification ${ }^{5}$.

En fait, les accords de Marrakech sont arrivés alors que les PAS avaient déjà déployé tous leurs effets dans la plupart des pays, en Afrique et en Amérique du

4. Mayer, citant d'autres auteurs, signale qu'au Mexique on n'a noté aucun mouvement important de ventes de terres ejidales, et qu'au Pérou les paysans étaient intéressés par la possibilité de recevoir un titre de propriété, mais ne souhaitaient pas dissoudre leur système de gestion communale. Il écrit: «Despite the neo-liberal push, the Fujimori government has quietly been using foreign aid to legalize land titles for comunidades as a priori before proceeding with titling individual properties within them» (Mayer 2002 : chap. 10).

5. Accord sur l'agriculture, article 15, par. 2.: «Les pays en développement membres auront la possibilité de mettre en œuvre les engagements de réduction sur une période pouvant aller jusqu'à dix ans. Les pays les moins avancés membres ne seront pas tenus de contracter des engagements de réduction. » 
Sud, et n'allaient pas tarder à le faire en Asie (l'entrée de la Chine dans l'OMC risque de toucher durement sa paysannerie). Les accords de Marrakech ont même conservé (ou introduit) de nombreuses règles (green box, mesures de sauvegarde, subventions aux intrants, investissements, etc. $\left.{ }^{6}\right)$ permettant aux pays du Sud de continuer à soutenir leurs paysanneries. Même l'Accord sur les ADPIC permet de poser des règles de dérogation à la contrainte des brevets en matière de nutrition, de santé publique, de développement socio-économique et de technologie, de plantes et d'animaux, ou en cas d'urgence nationale ${ }^{7}$.

Le problème vient de la difficulté à appliquer, ou simplement à transcrire au niveau national, un certain nombre d'éléments clés comme les subventions des intrants pour les petits paysans, la protection réelle de vastes bassins versants, de même qu'à prendre des mesures incitatives. Cette inaction n'est pas forcément due à un désintérêt des gouvernements, mais à leur adhésion à une doctrine qui peu à peu a démontré tous ses effets néfastes pour les paysans et les pays en général. Corrompus, achetés, brimés et menacés, parfois exécutés, les dirigeants et leurs organisations paysannes se sont tus progressivement, sauf dans les cas extrêmes des Paysans sans terre au Brésil ou des Indiens du Chiapas. On doit aussi être convaincu que beaucoup d'entre eux ont adhéré par désespoir à des guérillas (Colombie) ou des mouvements politiques et ethniques violents (Afrique centrale).

\section{LA PROTECTION DE LA BIODIVERSITÉ: ADPIC ET AIRES PROTÉGÉES}

Pour l'agriculture, la protection de la nature est venue comme une nouvelle donne, parfois clairement perturbatrice (il faut produire plus écologiquement), parfois comme un élément compensatoire, dans le cas de prestations liées au maintien de la biodiversité, des forêts, des aires humides, de la faune et de la flore en général. Cette constatation est valable surtout dans le Nord, où les nouvelles politiques agricoles ont intégré les éléments écologiques et les ont valorisés financièrement. Au Sud, le discours écologique a été quelque peu confondant: reproches de déboisement et de brûlis, mais sans compensations ou efforts de développement, incitation à valoriser les ressources biotiques, à les introduire dans le commerce, à les vendre à de grandes compagnies, mais finalement sans impact à vraiment large échelle (Bodyshop, Shaman Ind., Inbio). Par contre, ces pays ont subi de fortes pressions pour que les brevets des laboratoires du Nord sur le vivant soient homologués, et non copiés. Il est vrai que là aussi les conséquences ont été modestes en agriculture, à l'inverse du secteur pharmaceutique où les brevets ont empêché la production de médicaments bon marché et indispensables (rétrovirus).

Dans le domaine agricole, de nouvelles variétés résistantes ont été trouvées, mais leur application reste largement réservée aux climats tempérés, dont seule une petite minorité de pays du Sud, comme l'Argentine, fait partie ${ }^{8}$. Il n'est cependant pas exclu que des percées soient faites bientôt en ce qui concerne la

6. Accord sur l'agriculture, article 6 et annexe 2.

7. Articles 8 et 31 . Cf. le livre très complet de Correa (2000) sur les ADPIC et les pays en développement.

8. Les cultures transgéniques du Sud représentent en surface $26 \%$ du total en 2001. L'Argentine représente $22 \%$ du total mondial, plantés en soja, maïs et coton, la Chine 3\% (James 2001 : 6-8). 
banane, le manioc et d'autres plantes, et que changent dès lors les données du problème des relations agricoles entre les pays du Sud et les multinationales.

Certains organismes de recherche internationaux jouent parfois un jeu ambigu. Sous prétexte de libre mise à disposition des résultats de leurs recherches, ils livrent des génotypes variétaux du Sud à des laboratoires du Nord ${ }^{9}$. La recherche faite au Nord, si elle revient dans les pays d'origine, n'est alors plus disponible sans droits de brevet. Ces pratiques de biopiratage légalisé sont semble-t-il plus courantes qu'on ne le pense, bien que les chercheurs soient peu locaces sur le sujet, par crainte des licenciements. Il serait plus positif de développer la recherche biologique au Sud par les pays eux-mêmes, en créant des consortiums régionaux capables de rivaliser avec ceux du Nord, et en choisissant des lignes de recherche différentes, dans une perspective holistique de paysannat viable, qui remette en honneur l'approche par les systèmes de production (farming system) et l'utilisation à basse intensité d'intrants extérieurs.

\section{LES IMPACTS DE LA NOUVELLE POLITIQUE DE DÉVELOPPEMENT AGRICOLE}

Des statistiques officielles fiables et des études de terrain correctement menées dont nous disposons il ressort que deux effets apparemment contradictoires peuvent être observés depuis une vingtaine d'années:

๖ une amélioration générale moyenne de l'alimentation dans le Sud;

$\checkmark$ des poches de production, d'alimentation et de revenu agricoles déprimées dans beaucoup de zones rurales, surtout en Afrique et en Amérique centrale.

\section{$\square$ Amélioration de l'alimentation dans le Sud}

Il est indéniable que le nombre absolu de personnes sous-alimentées diminue régulièrement depuis 1990 . De 816 millions de personnes souffrant de faim en 1991, ce nombre est tombé à 717 millions en 1998. Il baisse de 6 millions par an, malgré une augmentation régulière de la population ${ }^{10}$. Mais les affamés augmentent dans l'Afrique subsaharienne, avec 168 millions de sous-nourris en 1991 et 194 millions en 1998. De même en Asie du Sud (de 289 millions à 303 millions) et au Moyen-Orient/Afrique du Nord (de 25 millions à 33 millions). L'Asie du Sud-Est et de l'Est connaît la plus nette amélioration (de 276 millions à 194 millions) (De Haen et al. 2001) ${ }^{11}$.

9. Le cas du yacon illustre des pratiques à la limite du biopiratage. En 2000, le Centre international de la pomme de terre (CIP), basé à Lima, livre des plants de yacon, un petit tubercule andin très riche en édulcorants, au Japon. Théoriquement il n'en avait pas le droit car ce matériel génétique n'avait pas encore été examiné et traité par le CIP. Mais les plants sont livrés d'abord à un organisme péruvien, qui les réexporte. En fait le yacon passe directement du CIP à l'aéroport de Lima. Dans les années précédentes, du yacon avait déjà été livré au Japon, qui en avait fait un «nouveau produit». Lorsque le Pérou, pays d'origine, avait demandé à disposer gratuitement de ces plants améliorés, il lui fut répondu que dorénavant ils étaient sous brevet. Les collaborateurs du CIP qui dénoncèrent l'affaire furent licenciés.

10. Cf. le rapport de la FAO The State of Food Insecurity in the World pour le Sommet mondial de l'alimentation + 1 (2001).

11. Le rapport de la FAO The State of Food Insecurity in the World souligne les progrès faits dans l'alimentation depuis 1969-1971. Le pourcentage de population consommant moins de 2100 calories par tête etpar jour a passé de 48,7\% en 1970 à seulement 6,2\% en 1999. Dans les pays du Sud, en moyenne, l'ingestion énergétique a passé de 2110 à $2800 \mathrm{kcal}$ par jour. 
Ainsi les régimes alimentaires s'améliorent-ils aussi, du moins au niveau de l'ingestion de calories. Dès les années 1990 le niveau moyen de 2000 calories par tête et par jour a été dépassé partout (sauf dans les régions de famines et de guerres). Des niveaux très satisfaisants de plus de 2400 calories sont notés sur tous les continents hormis l'Afrique. Pourtant, comme indiqué, ces chiffres ne sont que des moyennes; les extrêmes sont plus difficiles à estimer. On peut cependant penser que les couches favorisées dans le Sud ont à peu près le même régime calorique que dans le Nord (plus de 3000 calories), tandis que les couches défavorisées en sont au niveau énergétique africain moyen. Cette situation n'est donc nullement satisfaisante, et a été aggravée par les guerres, les PAS et autres avatars.

En outre, le niveau énergétique n'est pas tout. Il faut encore améliorer l'équilibre alimentaire, soit déplacer l'alimentation de produits pauvres en protéines vers d'autres plus nourrissants. Or, dans la majorité des zones rurales d'Afrique et d'Amérique du Sud la proportion de tubercules et de racines, ou féculents amidonnés, est prépondérante ou très importante. La proportion de produits carnés ou de dérivés du bétail est relativement faible, ce qui entraîne souvent des carences en micronutriments (De Haen et al. 2001 : 463). Une diversification alimentaire, que nous appelons transition alimentaire, est aussi nécessaire.

La relation entre alimentation et agriculture ne dépend pas seulement du niveau de production interne, mais des importations. Les classes riches et urbaines consomment plus d'aliments importés que les classes pauvres, elles consomment aussi des aliments plus riches en protéines «nobles» et plus d'aliments transformées par l'agro-industrie. En général cette tendance se remarque lorsque le pouvoir d'achat augmente, quel que soit le contexte.

Mais pour les masses rurales, et les pauvres urbains, cette transition alimentaire n'a pas encore eu lieu. Leur niveau de pauvreté requiert avant tout un accroissement quantitatif de l'alimentation et une stratégie d'amélioration diététique pour les groupes à risque (nourrissons, mères allaitant). Les plus sous-alimentés se trouvent dans les pays les plus agricoles (PNB agricole de $20 \%$ et plus), où l'agriculture représente une part de plus de $40 \%$ des exportations, et où la proportion de population rurale est de 75\% (De Haen et al. 2001 : 467, tableau 5). Dans ces pays, la proportion de sous-alimentés est d'environ $35 \%$. Dans ces cas-là, et dans tous les cas où la population rurale dépasse $50 \%$ du total, les efforts additionnels portés sur l'augmentation des cultures de rente pour l'exportation ne peuvent améliorer sensiblement la mauvaise alimentation locale, car les marchés d'importation sont éloignés, les produits vivriers importés trop chers et souvent inadaptés, et les prix des produits agricoles exportés en baisse réelle constante. C'est au contraire l'accent mis sur l'amélioration des cultures vivrières pour le marché de proximité et l'extension de ce dernier aux villes du pays qui peut accroître le revenu et le niveau alimentaire.

"Therefore growth of the agricultural sector is an essential component of reducing poverty and ensuring food security, given the dependence of the poor on the sector for their livelihoods » (De Haen et al. 2001 : 467).

\section{$\square$ L'appauvrissement des paysans}

Il est difficile dans beaucoup de cas de se rendre compte concrètement de l'appauvrissement des paysans, car la gamme des productions de substitution, mais 
aussi la pluriactivité, permettent apparemment de se nourrir et de vivre (vivoter) quel que soit le contexte économique général. En outre, nous le répétons, les études de terrain sérieuses sont quasi inexistantes depuis une vingtaine d'années.

Les apparences sont cependant trompeuses, car les stratégies d'adaptation résultent justement d'une aggravation des conditions de production et de vente. Les cas les plus flagrants concernent l'abandon par l'Etat du soutien aux cultures de rente, et en général à la paysannerie. Nous prendrons un exemple concret, celui de la cacaoyère au Cameroun.

Dans une étude ayant duré dix-huit mois, réalisée dans la région de MefouAfamba par Martin Ndonna (2001), l'attention s'est concentrée sur la substitution de production du cacao par le bananier plantain et le manioc, à la suite du PAS camerounais de 1990. Il ressort de ce travail que la production du vivrier a effectivement augmenté, pour la consommation des paysans, mais aussi pour la commercialisation vers Yaoundé. Cependant les recettes ne représentent que la moitié environ des ventes antérieures de cacao. Le complément est trouvé dans l'économie de cueillette et de taille (bois de chauffe) d'une part, et d'autre part dans la fabrication de vin et la distillation d'alcool de palme (odontol) de l'autre. Les effets de ces deux types de production complémentaires sont désastreux: grave atteinte à la biodiversité, et grave détérioration de la santé publique, surtout chez les jeunes. On ne peut donc voir dans cet exemple un succès de la reconversion forcée, même si à première vue il parait réjouissant de noter un retour au vivrier. Mais ce vivrier est de faible potentiel économique et calorique, il constitue une régression par rapport aux céréales. Dans ce cas précis, il faut noter que cette régression alimentaire concerne aussi le milieu urbain, où les élites ne peuvent plus avoir accès aux céréales importées.

Le cas de l'économie alimentaire de l'Albanie est encore plus parlant et dramatique, car il concerne un pays qui, avant la révolution de 1991, se nourrissait largement lui-même, et exportait même des fruits et légumes à ses voisins. Pour des motifs essentiellement idéologiques, il a été décidé par le gouvernement libéral de Sali Berisha d'abandonner la structure et les infrastructures productives existantes à leur triste sort, ou de les privatiser. Il était particulièrement poignant de voir les dernières truites d'une pisciculture abandonnée essayer de sauter par-dessus les bassins pour s'échapper. Mais il était encore beaucoup plus triste de voir pratiquement toutes les installations agro-industrielles saccagées et volées: immenses serres en verre éclatées au nord de Tirana, coopératives sans toit, machines dans les fossés, laiterie géante fermée près de Korça, canaux d'irrigation de la plaine centrale éventrés, culbutés, désaffectés. Sur les injonctions de la Banque mondiale, une pseudo-réforme agraire a divisé les coopératives et fermes d'Etat en parcelles de 1,5 hectare, une aberration économique (Auroi 1994; Auroi et al. 1994). Il est à signaler qu'aucun autre pays ex-communiste n'a heureusement fait une erreur pareille, mais l'Etat nouveau était aussi moins faible qu'en Albanie, et moins perméable aux discours soi-disant sociaux des organismes internationaux.

Le résultat de cette politique aventureuse s'exprime dans le fait que l'Albanie importe aujourd'hui (2000) la moitié de son blé, un tiers de sa viande et pratiquement tous les oléagineux. Ses paysans sont aussi obligés d'adopter des «stratégies alternatives» de tout type pour survivre, et une bonne part de l'économie mafieuse qui s'est installée tient à ces orientations idéologiques erronées. 


\section{L'AGRICULTURE, MOTEUR DU DÉVELOPPEMENT PAR LES EXPORTATIONS?}

La théorie des avantages compétitifs est devenue le credo de l'échange. Car l'avantage comparatif n'est qu'une apparence trompeuse de position forte sur le marché. Rien n'est immuable, et bientôt les Etats-Unis ou la Nouvelle-Zélande produiront de la laine de vigogne et s'imposeront sur les marchés. C'est là que réside la supériorité de l'avantage compétitif. Lorsqu'on est plus habile commercialement, avec de meilleurs réseaux et de forts capitaux, il est possible de concurrencer les denrées ou animaux de leurs pays d'origine. Il n'y a plus alors de centres d'origine à la Vavilovi2 ; il n'y a que des centres de dissémination (Harlan 198713), où les plus dotés en capitaux feront la loi.

Qu'est-ce que cela signifie pour les pays du Sud? Il est inutile de répéter qu'on leur vole leurs ressources, cela ne va pas faire reculer l'histoire. Dans une perspective de développement, il est plus judicieux d'envisager comment ces pays peuvent redevenir compétitifs.

Si l'on considère des exemples concrets, comme les camélidés andins importés par les Etats-Unis et d'autres pays, le riz basmati breveté aux Etats-Unis, le pisco péruvien accaparé par le Chili, la querelle entre Cuba et Baccardi concernant la marque, l'exclusivité de la France concernant le terme «champagne», l'emmenthal non reconnu comme spécifiquement suisse par Bruxelles, il apparaît que dans plusieurs de ces cas - pas tous - les détenteurs originaux ont fait preuve de paresse et d'excès de confiance. Les appellations auraient dû être déposées, des brevets pris dans plusieurs pays, la promotion du produit soutenue par l'Etat, etc. Sans doute n'est-il pas trop tard pour un grand nombre d'autres produits de terroir, mais pour avoir des exclusivités il faut une condition: le privé et l'Etat doivent collaborer.

De fait, le Chili, qui, dès le début des années 1980, a réussi cette osmose, montre le chemin. Ce succès repose sur deux organes essentiels: la Fundación Chile et Pro Chile. Leurs locaux se trouvent dans le bâtiment du Ministère du commerce et leurs portes sont largement ouvertes entre le public et le privé. La Fundación Chile réunit les producteurs pour discuter des normes de production pour l'exportation: types de produits, régulations sanitaires sur les principaux marchés, calibrage, etc. Pro Chile fait la promotion des produits sur les marchés extérieurs, et renvoie l'information concernant les tendances prédominantes. Les représentations de Pro Chile se trouvent souvent dans les locaux mêmes des ambassades. La percée agricole chilienne à l'exportation s'explique avant tout par des critères d'organisation et d'intégration, et non pas forcément par la dérégulation de l'économie (Auroi 1993, 1997).

Il y a donc un sérieux effort à faire dans les pays du Sud pour se doter d'infrastructures de promotion et de marketing sérieuses, et former le personnel requis.

12. Nicolaï I. Vavilov (1885-1943), botaniste russe, directeur de l'Institut national des plantes industrielles de Leningrad (Saint-Pétersbourg) entre 1930 et 1943. Il est l'auteur de la théorie des centres d'origine des plantes cultivées. Vavilov identifie huit centres d'origine, dont six dans les pays du Sud (Auroi 1992 : 59-60).

13. Jack Harlan complète la théorie de Vavilov sur les centres d'origine en expliquant que les centres de dissémination peuvent être plus vastes que les centres d'origine, car ceux-ci ne sont pas toujours exclusivement les centres de domestication des plantes ou animaux. 
Mais tout cela ne concerne qu'un aspect du problème, celui qui consiste à vouloir se battre sur le terrain défini par le marché mondial, par l'OMC. Il faut se poser la question de savoir si l'on pourrait offrir autre chose. On se penchera alors sur les marchés de niche, toujours dans l'agriculture, et sans doute on en trouvera. Mais le miel du Guatemala peut-il développer le Guatemala ? Là est la question, sachant que les termes de l'échange de toutes les denrées agricoles et naturelles se détériorent avec le temps, que les percées industrielles et informationnelles creusent chaque année le fossé. Ne doit-on pas alors concevoir que l'agriculture, comme secteur d'exportation, est forcément limitée?

Cela ne signifie nullement la fin de l'agriculture au Sud, mais sa réorientation. Celle-ci doit se faire vers le marché intérieur avant tout. Avec la croissance des villes, la demande est là, renforcée et modifiée par la transition alimentaire dans les milieux urbains, et donnant une nouvelle chance à l'agro-industrie.

\section{UNE NOUVELLE VISION POUR LE DÉVELOPPEMENT AGRICOLE ET RURAL: REGARDER VERS L'INTÉRIEUR}

Certes, la situation de la production et de la disponibilité en aliments n'est pas désastreuse en général, et nous pouvons être relativement confiants quant à la possibilité de nourrir une population croissante. Cependant les préoccupations ne concernent pas que la fonction alimentaire du monde rural, mais bien plus celles de la création d'emplois, de l'aménagement rural (habitat, services, infrastructures) et de la diversification des activités.

Il faut construire des stratégies alternatives, fondées sur d'autres critères que l'exportation à tout prix de produits agricoles dont la valeur baisse sans cesse sur les marchés internationaux. Ces stratégies alternatives devraient être basées sur le principe d' «utiliser nos propres ressources locales» (UNPRL), en anglais using our own local resources (UOOLR). Ce concept UNPRL, concept synthétique, signifie que si l'investissement pour le développement local ne veut pas venir à nous, nous allons à l'investissement. Investissement en argent, investissement en travail, en compétences, en échanges, en solidarités, en techniques et en matériaux.

Concrètement, cela veut dire qu'au lieu d'attendre que l'Etat intervienne, ce qu'il ne fait plus, il faut développer l'initiative locale. La plupart des travaux de construction locale (écoles, dispensaires, crèches, marchés, dépôts, aménagement de places, ponts) sont réalisables par les communautés rurales ellesmêmes. Le problème est de faire sauter la passivité ancestrale et de créer des consensus minimaux pour des projets nouveaux. Mais cet état d'esprit est maintenant relativement bien développé dans de nombreux pays. Il permet à un homme comme Toni Rüttimann ${ }^{14}$ d'avoir déjà construit des ponts suspendus pour plus de 150 communautés d'Amérique du Sud et du Cambodge. Pour des

14. Le jour de la remise de sa maturité (baccalauréat), à 19 ans, le jeune homme de Pontresina part en Equateur pour aider à la reconstruction suite à des inondations. Il va se lancer dans la construction de ponts piétonniers pendant quatorze ans, des ponts simples mais solides (garantis au moins trente ans), qui sont directement utiles à des communautés locales. Au Cambodge, c'est le premier ministre qui l'a fait venir, reconnaissant par là son extrême utilité pour le pays.

Cf. <www.iabse.ethz.ch/sei/backissues/abstracts.sei0001/BridgesMakingADifference.pdf>. 
sommes dérisoires, car les tubulures et câbles sont donnés par des entreprises, et le travail par les villageois. Lorsqu'une communauté demande un pont et est prête à faire l'effort, Rüttimann arrive, et le pont se fait en quinze jours. Il faut se demander pourquoi de tels exemples ne se multiplient pas dans d'autres domaines, ce qu'attendent les dirigeants du Sud et les ONG pour les diffuser largement. Avec un activisme de ce type, tous les pays du Sud auraient des infrastructures rurales minimales correctes en l'espace de vingt ans.

Les systèmes d'épargne-investissement locaux et régionaux devraient aussi progresser plus substantiellement et constituer des capitaux suffisants pour des ouvrages locaux. Mais pour cela il faut sortir de la vision «Grameen Bank» ${ }^{15}$, à trop petite échelle pour pouvoir être un moteur de développement. Non, il faut miser sur les capitaux locaux des gens riches qui en disposent, comme les marchands, et inciter ces derniers à investir dans leurs communautés.

L'important est donc de réfléchir maintenant au niveau local à des plans de développement minimaux réalisables sur dix ans, avec les intéressés. Le UNPRL devrait naturellement se constituer en mouvement dans chaque pays, avec certes des variantes nationales, mais en gardant l'objectif fondamental de couvrir systématiquement les besoins en infrastructures de zones entières, et que chaque village y participe.

Le rôle de l'Etat dans ce mouvement doit rester primordial. En tant que partenaires capables de dégager des capitaux importants, et en tant que garants du développement national, les Etats, à travers des organismes appropriés, doivent se concentrer sur les œuvres majeures: celles que l'on ne peut décemment réaliser à l'échelle locale - collèges, grands ponts, routes importantes, etc. Mais là aussi le concept UNPRL doit être appliqué. Les financements doivent être étudiés à partir des ressources fiscales et d'emprunts raisonnables, en évitant systématiquement l'endettement international et la complexification inutile des grands bureaux d'ingénieurs.

De même faut-il éviter le juridisme excessif, contraignant, en fait paralysant. Si l'on considère par exemple le problème de la propriété intellectuelle et du brevetage du vivant, les récents succès de l'Inde et du Brésil en matière de production de rétrovirus contre le sida montrent qu'il faut éviter ce piège, et savoir dire non. Car il est des principes qui viennent avant l'argent, et la santé en est un. Et l'alimentation en est un autre, ce qui veut dire que les semences doivent être accessibles à tous, et à bas prix. Les pays du Sud n'ont aucun avantage à accepter les règles contraignantes de 1'Accord sur les ADPIC, et ils doivent utiliser toutes les exceptions et dérogations permises.

Enfin, il faut revoir le modèle de croissance agricole, sortir de l'impasse des intrants chimiques ou fortement dépendants des hydrocarbures, pour étudier des systèmes plus «doux» et adaptés au contexte. Les nombreuses expériences de l'agriculture biologique devraient maintenant pouvoir être relayées par les Etats, comme veut le faire le Laos, pour lui donner une couverture plus large et une profondeur qu'elle n'a pas encore.

15. La Grameen Bank et d'autres expériences de ce type ne sont pas à dédaigner, mais ce ne sont pas quelques roupies données à des très pauvres femmes indiennes qui vont faire se développer les villages. On se situe plus ici dans une perspective assistancialiste. 
Le principe UNPRL ne veut nullement dire que le but ultime est l'autarcie, car les échanges ne peuvent et ne doivent pas être brimés, ils doivent être régulés. Il s'agit simplement de reconstruire les agricultures sur la base du principe de subsidiarité, ce qui signifie qu'avant de regarder qui peut résoudre ses problèmes, une communauté tente d'abord de les définir et de les résoudre elle-même. Il ne s'agit dès lors point du tout de vouloir tout faire soi-même, mais de dégager des ressources inventives parfois dormantes, ou dénigrées. Il est beaucoup de «traditions pour demain » qui sommeillent doucement, et que l'on devrait réveiller. De même existe-t-il nombre d'ingénieurs agronomes et d'autres professionnels dans le Sud qui conduisent des taxis, parce que personne ne les sollicite dans leur spécialité.

Un autre avantage du principe UNPRL est son lien étroit avec les soucis du développement durable. Compter sur ses propres ressources signifie chercher à minimiser les coûts énergétiques et en matières non renouvelables, et permettre la régénération des ressources renouvelables, matérielles et humaines. Au niveau de la psychologie sociale, la confiance collective et individuelle sera renforcée, dans certains cas créée à partir de zéro. Une fois bien assise, elle donnera une force sociale et politique capable de discuter enfin d'égal à égal avec les instances de décision supérieures et les partenaires travaillant en réseau.

\section{L'APPORT EXTÉRIEUR}

Répétons-le: les apports extérieurs, à tous les niveaux, doivent être complémentaires, et non substitutifs. Ils ne doivent pas consister en un oreiller de paresse pour l'initiative locale, mais en un appoint indispensable pour réaliser un plan ou un projet. Ce principe implique que les coopérations internationales n'échafaudent plus aucun projet pour des populations locales, mais qu'elles se tiennent prêtes à répondre à des demandes ${ }^{16}$. Ces demandes peuvent émaner d'organismes gouvernementaux et de collectivités locales, voire de groupes privés à vocation économique et sociale. L'important dans la démarche réside cependant dans le fait que la demande soit pour l'essentiel élaborée par les capacités locales et basée au maximum sur elles. Ce sont des locaux et des nationaux qui doivent formuler les demandes, pas des expatriés agissant comme conseillers techniques. Ceux-ci ne devraient intervenir que dans un deuxième temps.

Cependant, même dans ce cas de figure un peu idéalisé, il reste que les règles du jeu sont fixées par l'extérieur, par l'appoint décisif. Il est vrai que s'il y a partenariat, il y aura et il devra toujours y avoir échange d'opinions. L'important est surtout de créer un climat dans lequel les partenaires connaissent le principe UNPRL et respectent le principe de subsidiarité. Les communautés savent généralement très bien quels sont leurs besoins, mais ceux-ci ne correspondent que rarement aux visions de la coopération technique. Actuellement, les organes internationaux, suivis par les coopérations nationales, ont fixé quatre objectifs pour le développement agricole:

16. Dans son article sur le pastoralisme dans la Corne de l'Afrique, Baxter (1994) montre bien comment les technocrates veulent absolument faire du ranching à la place du semi-nomadisme, et fixent tous les objectifs pour les pasteurs eux-mêmes. 
- accès aux marchés ;

- sécurité alimentaire;

- lutte contre la pauvreté ;

- protection de l'environnement.

Tout d'abord, la sécurité alimentaire devrait constituer l'objectif prioritaire, et l'accès aux marchés (sous-entendu internationaux) venir en dernier lieu. Ensuite, la lutte contre la pauvreté ne peut être envisagée sous l'approche habituellement définie des filets de sécurité, aide d'urgence et accent sur les groupes cibles. La pauvreté ne peut être diminuée que par une croissance agricole généralisée, comme le montre l'analyse statistique de ces trente dernières années. Enfin, la protection de l'environnement ne doit pas être conçue comme un frein au développement agricole, mais dans une approche symbiotique entre l'homme et la nature.

Cependant, cette démarche n'est pas suffisante si le développement rural et agricole n'est pas revu dans son essence. Il ne sert à rien de fixer des domaines d'action, des principes méthodologiques et d'opération si ceux-ci sont au service d'une mauvaise cause. En agriculture et en alimentation, le principe UNPRL doit être appliqué :

๖ produire et se nourrir le plus possible localement, aux niveaux national et régional ;

- construire les réseaux (physiques et de communication) du futur pour nourrir les villes à partir de la production nationale;

• taxer les importations alimentaires à un niveau qui permette le développement de la production nationale, voire régionale, selon le principe de la sécurité alimentaire;

- appliquer les principes du développement durable à l'utilisation des ressources biotiques et minérales;

• utiliser l'énergie et les intrants locaux, et adapter les technologies à cette fin ;

a viser la transformation des produits bruts sur place, et donner à ces industries agroalimentaires à petite échelle des priorités et des niveaux de promotion adéquats ;

• accroître ou créer des institutions d'épargne locale pour l'investissement local;

- exporter, s'il y a des marchés complémentaires ou de niche, selon les principes du commerce équitable et de l'agriculture biologique.

L'aide internationale publique à l'agriculture n'a pas cessé de décliner pour les secteurs «classiques» depuis 1990, mais elle a légèrement augmenté pour la protection de l'environnement, l'agro-industrie et les infrastructures ${ }^{17}$. D'une manière générale, par rapport à l'aide publique au développement totale, la part de l'agriculture a augmenté de $17 \%$ à $20 \%$ entre 1990 et 1998 . Sa continuation,

17. Secteurs classiques: sols et eau, recherche, formation et vulgarisation, intrants, services agricoles, production végétale, élevage, pêche, foresterie, autres. Diminution de l'aide de 11 milliards à 8 milliards de dollars. Autres secteurs: production d'intrants, protection de l'environnement, agro-industrie, développement rural et régional, systèmes hydrauliques. Augmentation de 3,8 milliards à 5,6 milliards de dollars (De Haen et al. $2001:$ 472, tableau 10). 
voire son augmentation devraient cependant être largement revues selon les principes d'une utilisation plus intensive des ressources locales destinées aux marchés de proximité, dans le respect du développement durable, et d'un réel empowerment des groupes de population.

\section{BIBLIOGRAPHIE}

Aly Bâ Mamadou, 2001, «Les difficultés de l'insertion: la production du haricot vert au Burkina Faso», in Auroi C. et Schümperli Younossian C. (dir.), Le commerce durable. Vers de plus justes pratiques commerciales entre le Nord et le Sud, Genève; IUED, pp. 41-56.

Auroi C., 1992, La diversité biologique. La vie en péril, coll. Les Dossiers de l'environnement, Genève: Georg.

—, 1993, «Le cas du Chili: essai de croissance vers l'extérieur», in Etienne G. et Revel-Mouroz J. (dir.), Economies d'Asie et d'Amérique latine: changements de cap, Genève: Olizane.

—, 1997, «Effets de l'ouverture du commerce mondial sur la restructuration agraire: exemples de la Bolivie et du Chili», in Haubert M. (dir.), Les paysans, l'Etat et le marché. Sociétés paysannes et développement, Paris: Publications de la Sorbonne, pp. 97-110.

- (dir.), 1994, A Development Strategy for the Mountain Areas of Albania. Final Report, vol. 1 and 2, IUED, May, $19 \mathrm{p}+158 \mathrm{p}$.

Auroi C., Egger U., Zehnder R. et Moos F., 1994, Implementation of a Development Strategy for the Mountain Areas of Albania. Report on the Mission of September 1994, IUED, $29 \mathrm{p}$.

Auroi C. et Maurer J.-L., 1998, «Tradition et modernisation des économies rurales: Asie - Afrique - Amérique latine», Mélanges en l'honneur de Gilbert Etienne, Genève/Paris: IUED/PUF, 1998.

Barkin D., 2000, Estrategias de los campesinos mexicanos: alternativas frente a la globalizacion, <http://136.142.158.105/LASA98/BARKIN.PDF>.

Barkin D. et Suárez B., 1982, El fin de la autosuficiencia alimentaria, México: Nueva Imagen.

Baxter P.T.W., 1994, «From Telling People to Listening to Them: Changes in Approaches to the Development and Welfare of Pastoral Peoples », in Salih M., Inducing Food Insecurity: Perspectives on Food Policies in Eastern and Southern Africa, Uppsala: The Nordic Africa Institute.

Bessis S., 1985, L'arme alimentaire, Paris: La Découverte, 1985.

BIT, 1999, Réformes économiques, emploi et rôle des partenaires sociaux en Afrique de l'Ouest francophone. Pour un renforcement du dialogue social, Séminaire de Dakar, 27-29 avril 1998, Dakar: BIT.

Cardoso F.H. et Faletto E., 1978, Dépendance et développement en Amérique latine, coll. Politiques, Paris : PUF.

Correa C.M., 2000, Intellectual Property Rights, the WTO and Developing Countries: The TRIPs Agreement and Policy Options, London: Zed Books.

De Haen H., Stamoulis K. et Broca S., «Reducing Hunger and Poverty through Agricultural Growth: Dealing with Key Challenges », Nord-Süd Aktuell (Hamburg), nº 3, pp. 461-473.

Feder E., 1978, Capital financiero y descomposicion del campesinado, Bogota: Punta de Lanza.

Gunder Frank A., 1970, Le développement du sous-développement, l'Amérique latine, Paris: Maspero, 1970.

Harlan J., 1987, Les plantes cultivées et l'homme, Paris: ACCT; Conseil international de la langue française.

Haubert M. (dir.), 1997, Les paysans, l'Etat et le marché. Sociétés paysannes et développement, Paris: Publications de la Sorbonne.

James C., 2001, «Global Review of Commercialized Transgenic Crops : $2001 »$, ISAAA Briefs, $\mathrm{n}^{\circ} 24$.

Mayer E., 2002, The Articulated Peasant: Household Economies in the Andes, Boulder (Colorado): Westview Press, 2002.

Ndonna M., Du cacaoyer aux cultures vivrières. Contribution des cultures vivrières dans l'amélioration des revenus de la paysannerie de la zone cacaoyère camerounaise. Cas du manioc et du bananier plantain dans le département de Mefou et Afamba: limites et perspectives, mémoire de diplôme de recherche, Genève: IUED, 2001. 